\title{
Topological phase transition and charge-spin coupling in the kagome metal YMn6Sn6
}

\section{Peter Siegfried ( $\nabla$ psiegfri@gmu.edu )}

George Mason University https://orcid.org/0000-0002-0145-2899

\section{Hari Bhandari}

George Mason University

D Jones

George Mason University

\section{Madhav Ghimire}

Tribhuvan University

\section{Rebecca Dally}

NIST

\section{Poudel}

National Institute of Standards and Technology https://orcid.org/0000-0003-0865-0385

\section{Bleuel}

National Institute of Standards and Technology

\section{Jeffrey Lynn}

National Institute of Standards and Technology https://orcid.org/0000-0003-3626-4932 Igor Mazin

George Mason University https://orcid.org/0000-0001-9456-7099

\section{Nirmal Ghimire}

George Mason University https://orcid.org/0000-0002-8474-4968

\section{Article}

Keywords: Fermi surface, topological phase transition, magnetism and electronic topology

Posted Date: September 7th, 2021

DOl: https://doi.org/10.21203/rs.3.rs-828140/v1

License: (1) This work is licensed under a Creative Commons Attribution 4.0 International License. Read Full License 
Version of Record: A version of this preprint was published at Communications Physics on March 15th, 2022. See the published version at https://doi.org/10.1038/s42005-022-00833-2. 


\section{Topological phase transition and charge-spin coupling in the kagome metal $\mathrm{YMn}_{6} \mathrm{Sn}_{6}$}

Peter E. Siegfried ${ }^{1,2 *}$, Hari Bhandari ${ }^{1,2}$, David C. Jones ${ }^{1,2}$, Madhav P. Ghimire ${ }^{3,4}$, Rebecca L. Dally $^{5}$, Lekh Poudel $^{5,6}$, Markus Bleuel ${ }^{5,6}$, Jeffrey W. Lynn ${ }^{5}$, Igor I. Mazin ${ }^{1,2}$, Nirmal J. Ghimire ${ }^{1,2 \dagger}$

${ }^{1}$ Department of Physics and Astronomy, George Mason University, Fairfax, VA 22030, USA.

${ }^{2}$ Quantum Science and Engineering Center, George Mason University, Fairfax, VA 22030, USA.

${ }^{3}$ Central Department of Physics, Tribhuvan University, Kirtipur, Kathmandu 44613,, Nepal.

${ }^{4}$ Leibniz Institute for Solid State and Materials Research, IFW Dresden, Helmholtzstr. 20, D01069 Dresden, Germany.

${ }^{5}$ NIST Center for Neutron Research, National Institute of Standards and Technology, Gaithersburg, MD 20899, USA.

${ }^{6}$ Department of Materials Science and Engineering, University of Maryland, College Park, MD 20742, USA.

The Fermi surface (FS) is essential for understanding the properties of metals. It can change under both conventional symmetry-breaking phase transitions and Lifshitz transitions (LTs), where the FS, but not the crystal symmetry, changes abruptly. Magnetic phase transitions involving uniformly rotating spin textures are conventional in nature, requiring strong spinorbit coupling (SOC) to influence the FS topology and generate measurable properties. LTs driven by a continuously varying magnetization are rarely discussed. Here we present two

*psiegfri@gmu.edu

†'nghimire@gmu.edu 
such manifestations in the magnetotransport of the kagome magnet $\mathbf{Y M n}_{6} \mathrm{Sn}_{6}$; one caused by changes in the magnetic structure and another by a magnetization-driven LT. The former yields a $10 \%$ magnetoresistance enhancement without a strong SOC, while the latter a $45 \%$ reduction in the resistivity. These phenomena offer a unique view into the interplay of magnetism and electronic topology, crucial for understanding the rare-earth counterparts, such as $\mathbf{T b M n}_{6} \mathbf{S n}_{6}$, recently shown to harbor novel correlated topological physics.

Conventionally, a phase transition is marked by the onset of an order parameter due to a spontaneously broken symmetry. Typically the order parameter is related to some thermodynamic quantity, e.g. magnetization, density, or polarization, that grows continuously after the transition. An LT, on the other hand, is a unique electronic transformation in metals associated with a change in the FS topology ${ }^{1}$ that occurs without breaking any symmetries, and as such, LTs driven by a continuously changing magnetization are quite rare. The effect, however, is enhanced with the lowering of the effective dimension of the FS pocket involved and can play an important role in stabilizing new types of topological phases $^{2-4}$. In contrast, conventional field induced transitions concerning changes in magnetic texture (1st or 2 nd order) involve a uniform rotation in spin space. Their effect on the electronic structure, and therefore on the transport properties, is controlled solely through the relativistic spin-orbit interaction, which is weak, determined by the fine structure constant $\alpha \simeq 1 / 137$, but grows with atomic number, $Z$. Typically a strong SOC is required to observe magnetotransport signatures in this case. Here we show that the kagome lattice magnet $\mathrm{YMn}_{6} \mathrm{Sn}_{6}$ is a prototype system in terms of electronic transport that shows the two above mentioned magnetotransport properties. First, it exhibits a magnetization driven LT, with a strong effect on 
the $c$-axis conductivity, and second, a sizable electron-spin coupling without a strong SOC.

$\mathrm{YMn}_{6} \mathrm{Sn}_{6}$ is a ternary kagome magnet, a family of materials known to host a variety of topological states and phenomena ${ }^{5-8}$. It crystallizes in the hexagonal space group P6/mmm (\#191) with Mn atoms forming a kagome net in the basal plane as depicted in Fig. 1(a,b). The material orders antiferromagnetically at $340 \mathrm{~K}$ and quickly transitions into an incommensurate spiral below $333 \mathrm{~K}$. At all temperatures, a magnetic field applied along the $c$-axis cants the moments lying in the $a b$-plane towards the $c$-axis, while for a magnetic field applied in the $a b$-plane, a series of competing phases ${ }^{10-12}$ are formed, as depicted in Fig. 1(c) (see SI2 for details). A schematic plot of the different magnetic structures and their wavevectors is shown in Fig. 1(d). Application of a magnetic field in the $a b$-plane first cants the spins rotating in the $a b$-plane, creating a distorted spiral (DS) phase, characterized by the wavevector $\mathbf{k}=(0,0,0.26)$. When the magnetic field reaches $H_{1}$, the spins flop to the perpendicular plane and cant towards the direction of the external field, maintaining the incommensurate $c$-axis spiral with only a slight increase in the spiral wavevector $\left(k_{z}=0.27\right)$. This phase is a transverse conical spiral (TCS). When the field reaches $H_{2}$, the spins flop back into the $a b$-plane forming a commensurate fan-like (FL) phase. A projection of the FL spins onto the direction of the magnetic field (x-component) and along the perpendicular direction in the $a b$-plane results in a spin pattern repeating every two and four unit cells respectively (see Fig. SI3), producing two different commensurate Bragg peaks observed in neutron diffraction at $k_{z}=$ 0.5 and 0.25 , as shown schematically by the red lines in Fig. 1(d). At $H_{3}$ the moments saturate to form the forced ferromagnetic (FF) phase. 
Magnetoresistance (MR) at $1.8 \mathrm{~K}$ is plotted on the left axes of Figs 2(a) and (b). To compare the MR with the underlying magnetism, corresponding magnetization data are plotted in each figure on the right axis (see SI3 for the sensitivity of resistivity to the magnetic ordering with temperature). For the field applied in the $a b$-plane presented in panel (a), magnetization (black) shows a spin-flop transition at 2.2 $\mathrm{T}\left(H_{1}\right)$ corresponding to the DS-TCS phase transition and a slope change slightly above $6.5 \mathrm{~T}\left(H_{2}\right)$ where the TCS-FL phase transition occurs. When current is applied in the $a b$-plane ( $\left.\mathrm{MR}_{I_{a b}}\right)$ (blue), the MR shows a small jump at $2.2 \mathrm{~T}\left(H_{1}\right)$, a small slope change at $4.5 \mathrm{~T}$, and a sharp drop quickly above $8 \mathrm{~T}$. The decrease in $\mathrm{MR}_{I_{a b}}$ above $8 \mathrm{~T}$ can be understood as polarization of the FL spins in the direction of the magnetic field, reducing spin fluctuations and the electron scattering. The slope change at $4.5 \mathrm{~T}$ has no corresponding change in the magnetization and is a small feature in this measurement configuration. In contrast, the MR with current along $c\left(\mathrm{MR}_{I_{c}}\right)$ (red) shows three remarkable features. First, there is no noticeable jump at $H_{1}$ despite the magnetic phase transition with a significant increase in magnetization. Second, $\mathrm{MR}_{I_{c}}$ drops sharply at $4 \mathrm{~T}$ with no corresponding change in magnetization. Third, it again increases sharply, by about $10 \%$ at $6 \mathrm{~T}$, peaks at $H_{2}$, and then again drops sharply.

MR and magnetization with the magnetic field along the $c$-axis are presented in Fig. 2(b). Here, the magnetization smoothly increases up to $9 \mathrm{~T}$ indicating a continuous canting of the spins towards the $c$-axis. MR for current in the $a b$-plane $\left(\mathrm{MR}_{I_{a b}}\right)$ (green) initially increases, reaching a maximum at $4.5 \mathrm{~T}$ and then decreases with a smaller overall change $(\sim 8 \%)$ in $\mathrm{MR}$. The drop for MR with the current applied along the $c$-axis $\mathrm{MR}_{I_{c}}$ (orange) is much larger (45\%) beginning at $5 \mathrm{~T}$. We refer to this magnetic field, where MR drops without a corresponding change in magnetization, 
as $H^{\prime}$.

In Fig. 2 (c), the MRs measured in all four configurations at $1.8 \mathrm{~K}$ are plotted together as a function of the corresponding magnetic moment. The plot shows that the $H^{\prime}$ MR drop occurs, irrespective of orientation, when the magnetization reaches $6 \mu_{B} F . U .^{-1}$, indicating that the $H^{\prime} \mathrm{MR}$ drop is magnetization induced. The highly anisotropic nature of this $H^{\prime}$ feature, indicated by a larger change for current along the $c$-axis than in the $a b$-plane, suggests that the electronic state driving it is highly anisotropic. It is to be noted that the small jump in $\mathrm{MR}_{I_{c}}$ with field in the $a b$ plane (red curve) at $1 \mu_{B} F . U .^{-1}$ of $M$ is related to the sudden increase in $M$ at $H_{1}$. The temperature evolution of the $\mathrm{MR}_{I_{c}}$ for the in-plane field is depicted in Fig. 2(d) for selected temperatures. The $H^{\prime}$ drop in MR starts broadening at $20 \mathrm{~K}$ and disappears above $80 \mathrm{~K}$. The $H_{2}$ MR peak follows the FL phase shown in the phase diagram in Fig. 1(c). The peak persists to $150 \mathrm{~K}$ and disappears by $200 \mathrm{~K}$, matching with the FL phase boundary at $170 \mathrm{~K}^{10}$. The full temperature range measured for each of the current and applied field configurations is shown in Fig. SI5.

To relate the MR features with the microscopic magnetic structure, we plot the $1.8-\mathrm{K} \mathrm{MR}_{I_{c}}$ for the in-plane magnetic field together with the magnetic Bragg peak measured using small angle neutron scattering at $4 \mathrm{~K}$ in Fig. 3(a). The intensity of the Bragg peak does not show a noticeable change below $H_{1}$. At $H_{1}$, the Bragg peak loses intensity and the wavevector slightly increases marking the DS-TCS phase transition. There is no apparent change in the intensity or the wavevector at $H^{\prime}$, the MR turnover field, signifying no change in magnetic structure at $H^{\prime}$ besides a gradual spin canting. The $H_{2}$ peak in MR corresponds exactly to the region where the wavevector of the 
magnetic Bragg peak changes at the TCS-FL phase boundary. Once in the FL phase, the MR starts decreasing as the magnetic field starts canting the spins along the field. These observations unambiguously show that the $H^{\prime}$ MR drop and $H_{2}$ MR peak are associated with the magnetic field induced changes in the electronic and magnetic structures, respectively, with the former being magnetization driven indicating an LT. An LT is usually accompanied by a change in carrier concentration observable through Hall effect measurements. However, we do not see a noticeable slope change in the Hall conductivity [Fig. 3(b)] or resistivity (Fig. SI7) at $H^{\prime}$, but rather a gradual change. This paradox can be related to the complex fermiology of a metal with a complicated band structure (see SI5, and Fig. SI9).

To investigate the microscopic origin of the LT, we calculated the non-relativistic electronic band structure in the experimental zero-field zero-temperature spiral magnetic phase and in the fully polarized ferromagnetic state. In the zero-field state (blue bands), there are no bands at the Fermi energy $\left(E_{F}\right)$ at the $L$-point as depicted in Fig. 4(a). On the contrary, in the polarized state, a flat band from the spin majority channel appears at $E_{F}$, emphasized in bold in Fig. 4(a). It forms a highly anisotropic hole pocket around $L$, visible as a pancake shape in Fig. 4 (b), where the Fermi surface for the spin majority bands is depicted. This hole pocket is highly dispersive along $c$, nearly flat in the $L-A$ direction and rather heavy along $L-H$. Due to its quasi-1D nature, this hole pocket contributes mainly to the transport along $c$. Other features of the Fermi surface include one large electron pocket around $H$ and one large hole pocket around $K$, forming two networks of interconnected triangular pillows. These pockets are also more dispersive along $c$, but are much less anisotropic than the hole pocket around $L$. In addition, there are also two small 
electron pockets: one between $L$ and $M$, a remnant of a quasi-2D band, and another around $\Gamma$. Overall, somewhat counterintuitively for the layered structure of $\mathrm{YMn}_{6} \mathrm{Sn}_{6}$, the spin-up electrons propagate more easily perpendicular to the Mn planes. Spin-down bands (not shown), on the contrary, prefer the in-plane transport. It can be inferred that the new Fermi surface appears, via an LT, well below full saturation, i.e., when the moments attain a value of $M=6 \mu_{B} F \cdot U .^{-1}$ at $H^{\prime}$, and is responsible for the sharp drop in MR. This is consistent with the fact that the drop occurs at different field magnitudes for the in-plane and out-of-plane field directions, but at the same $M$, and is considerably more pronounced for the $c$-axis MR.

Turning attention to the $\mathrm{H}_{2}$-MR peak that occurs during the TCS-FL phase transition, we calculate the hopping probabilities (which in turn determine the conductivities) in each of the phases within a tight-binding approximation. For a 1D chain, the probability for an electron to travel along the chain is determined by the lowest of the individual probabilities which we call the bottleneck. The hopping between two electrons with non-parallel spins is proportional to half of the cosine of the angles between them. The experimentally determined largest angles between two spins in the TCS and FL phases are $67.9^{\circ}$, and $81^{\circ}$, respectively ${ }^{11}$ (see SI6 for details). Thus, the bottleneck factor in the TCS and FL phases for the $c$-axis transport is 0.83 , and 0.76 , respectively. The bottleneck factor along $c$ (but not in-plane) in the TCS phase is $9 \%$ higher than that in the FL phase, quantitatively accounting for the $10 \%$ rise of the interplaner magnetoresistance. It is to be noted here that this effect is entirely due to the spin texture and not to the SOC, as evidenced by a negligible MR for a much larger magnetization change at $H_{1}$. Such an interplaner MR has been discussed with respect to the chiral solition lattice phase in $\mathrm{Cr}_{1 / 3} \mathrm{NbS}_{2}{ }^{13}$, where the Dzyaloshinskii- 
Moriya interaction driven chirality is inherently coupled to the SOC. The charge-spin coupling in $\mathrm{YMn}_{6} \mathrm{Sn}_{6}$ is unique in that it requires neither chirality nor a strong SOC.

Common LTs, driven by doping, strain, or pressure, are often associated with van Hove singularities ${ }^{14}$ that are difficult to observe via transport measurements. Moreover, those LTs are rather inflexible, since chemical doping cannot be controlled in situ within the same sample, and varying strain or pressure appreciably is difficult. An uncommon magnetization driven LT is free of these shortcomings, and has potential as a tool to tune the FS topology. We emphasize that while this specific LT is rather fortuitous, considerable modification of the FS via applied field in spiral magnets is to be expected, and it is likely that similar LTs will be discovered in other spiral magnets. The magnetic transition at $H_{2}$, which occurs between two ordered phases, as opposed to the spinflop transition at $H_{1}$, does affect the MR (but only along $c$ ), as shown in Fig. 2(a). The scale of this effect can be estimated from the standard formula relating the one-electron hopping probability to the angle between the local magnetic moments ${ }^{16}$ and is consistent with the experimental change in MR. This behavior marks the electron-spin interaction without the requirement of strong SOC or spin chirality, as in the chiral magnets ${ }^{13,15}$.

In summary, our magnetotransport measurements in varying applied magnetic fields, and in different current directions, uncover two nontrivial effects in magnetoresistance. Using first principles calculations, we were able to identify one of them, which occurs at a magnetization of $M=6 \mu_{B} F \cdot U .^{-1}$ for any field direction, as a magnetization driven Lifshitz transition, qualitatively different from common pressure or strain driven ones. The other effect demonstrates how 
magnetotransport phenomena are intimately related to the change in magnetic texture. These types of effects are strongly desired for potential spintronic applications and, thus, our results provide a compelling observation to stimulate new theoretical and experimental research into the interaction between the topology of electronic bands in kagome lattices, magnetic interactions, and spin-orbit coupling, especially in the rare-earth ternary kagome magnet systems.

\section{Methods.}

Single Crystal Growth. Single crystals of $\mathrm{YMn}_{6} \mathrm{Sn}_{6}$ were grown by the self-flux method. Y pieces (Alfa Aesar; 99.9\%), Mn pieces (Alfa Aesar; 99.95\%), and Sn shots (Alfa Aesar; 99.999\%) were loaded in a 2-ml aluminum oxide crucible in a molar ratio of 1:1:20. The crucible was sealed in a fused silica ampoule under vacuum and heated to $1175^{\circ} \mathrm{C}$ over 10 hours, homogenized at 1175 ${ }^{\circ} \mathrm{C}$ for 12 hours, and then cooled to $600{ }^{\circ} \mathrm{C}$ over 100 hours. Upon reaching $600{ }^{\circ} \mathrm{C}$ the excess flux was decanted from the crystals using a centrifuge leaving behind well-faceted hexagonal crystals up to $100 \mathrm{mg}$ in mass. All measurements were performed on crystals oriented for the $[0,0,1]$ and $[1,1,0]$ directions.

Transport and Magnetization. For electrical transport measurements samples were polished to dimensions of approximately $1.00 \times 0.40 \times 0.15 \mathrm{~mm}$ with the long axis corresponding to either the $c$-axis, or the in-plane, $[1,1,0]$, direction. An excitation current of $4 \mathrm{~mA}$ was used in all measurements presented. Identical data were also measured with a $2 \mathrm{~mA}$ excitation, however the higher $4 \mathrm{~mA}$ current produced less noise without impacting the features observed. Electrical contacts 
were affixed using Epotek H20E silver epoxy and $25 \mu \mathrm{m}$ Pt wires with typical contacts resistances of $\approx 20 \Omega$, such that current was directed along the $c$-axis or in the $a b$-plane. MR and Hall effect data were collected using the resistivity option and rotator within a Quantum Design DynaCool PPMS equipped with a 9 T magnet. MR is defined as $\mathbf{M R}=(\rho(H)-\rho(0)) / \rho(0)$, where $\rho(H)$ and $\rho(0)$ are the longitudinal resistivity with and without an applied magnetic field, $H$. The Hall resistivity was asymetrized from the positive and negative applied magnetic fields via $\rho_{x z}=\left[\rho_{T}(+H)-\rho_{T}(-H)\right] / 2$, where $\rho_{T}$ is the resistivity measured via the transverse voltage contacts in the Hall bar geometry. Hall conductivity for current along the $c$-axis was calculated via the relation $\sigma_{z x}=\rho_{x z} /\left(\rho_{x z}^{2}+\rho_{z z}^{2}\right)$ where $\rho_{z z}$ is the longitudinal resistivity for current along the $c$-axis and field applied in the $a b$-plane, and $\rho_{x z}$ is the Hall resistivity for current along $c$ and field applied in the $a b$-plane. DC magnetization measurements were performed in the same PPMS system using the ACMS II option.

Small Angle Neutron Scattering. Small Angle Neutron Scattering data were collected with the NG-7 SANS instrument at the NIST Center for Neutron Research using a $9 \mathrm{~T}$ horizontal field magnet $(H \|[1,1,0])$ and in the temperature range from $4 \mathrm{~K}$ to $300 \mathrm{~K}$. For Fig. 3(a), conversion of wavevector to $\AA^{-1}$ from r.l.u units is made through the following relation, $\mathbf{Q}\left(\AA^{-1}\right)=2 \pi L / c$, where $L$ is the $z$-component of the wavevector in r.l.u. $[\mathbf{k}=(H, K, L))]$, and $c$ is the $c$-axis unit vector length in $\AA$.

First-principles Calculations. Most calculations were performed using the projected augmented wave pseudo-potential code $\operatorname{VASP}^{17}$ and the gradient-dependent density functional of reference 
(18). For control purposes, some calculations were also repeated using the all-electron linearized augmented plane wave code WIEN $2 \mathrm{k}^{19}$.

Data availability. The authors declare that the main data supporting the findings of this study are available within the article and its Supplementary Information files. Extra data are available from the corresponding author upon request.

\section{References.}

1. Lifshitz, I. M. Anomalies of electron characteristics of a metal in the high pressure region. Soviet Physics JETP 11, 1130-1135 (1960).

2. Volovik, G. E. Exotic Lifshitz transitions in topological materials. Physics-Uspekhi 61, 89 (2018).

3. Okada, Y. et al. Observation of Dirac Node Formation and Mass Acquisition in a Topological Crystalline Insulator. Science 27, 1496-1499 (2013).

4. Zeljkovic, I. et al. Mapping the unconventional orbital texture in topological crystalline insulators. Nature Physics 10, 572-577 (2014).

5. Villars, P. $\mathrm{YMn}_{6} \mathrm{Sn}_{6}$ Crystal Structure Springer Materials sd_1804357 (2016).

6. Kitaori, A. et al. Emergent electromagnetic induction beyond room temperature. arXiv preprint arXiv:2103.02814 (2021). 
7. Li, M. et al. Dirac cone, flat band and saddle point in kagome magnet $\mathrm{YMn}_{6} \mathrm{Sn}_{6}$. Nature Communications 12, 1-8 (2021).

8. Yin, J.X. et al. Quantum-limit Chern topological magnetism in $\mathrm{TbMn}_{6} \mathrm{Sn}_{6}$. Nature $\mathbf{5 8 3}, 533-$ $536(2020)$.

9. Zhang, H. et al. Topological magnon bands in a room-temperature kagome magnet. Physical Review B 101, 100405 (2020).

10. Ghimire, N. J. et al. Competing magnetic phases and fluctuation-driven scalar spin chirality in the kagome metal $\mathrm{YMn}_{6} \mathrm{Sn}_{6}$. Science Advances 6, eabe2680 (2020).

11. Dally, R. L. et al. Chiral properties of the zero-field spiral state and field-induced magnetic phases of the itinerant kagome metal $\mathrm{YMn}_{6} \mathrm{Sn}_{6}$. Physical Review B 103, 094413 (2021).

12. Wang, Q. et al. Field-induced topological Hall effect and double-fan spin structure with a c-axis component in the metallic kagome antiferromagnetic compound $\mathrm{YMn}_{6} \mathrm{Sn}_{6}$. Physical Review B 103, 014416 (2021).

13. Togawa, Y. et al. Interlayer magnetoresistance due to chiral soliton lattice formation in hexagonal chiral magnet $\mathrm{CrNb}_{3} \mathrm{~S}_{6}$. Physical review letters 111, 197204 (2013).

14. Sunko, V. et al. Direct observation of uniaxial stress-driven Lifshitz transition in $\mathrm{Sr}_{2} \mathrm{RuO}_{4}$. npj Quantum Materials 4, 1-7 (2019).

15. Neubauer, A. et al. Topological Hall Effect in the A Phase of MnSi. Physical Review Letters 102, 186602 (2009). 
16. Khomskii, D. I. Basic Aspects of the Quantum Theory of Solids: Order and Elementary Excitations. Cambridge University Press (2010).

17. Kresse, G. \& Furthmüller, J. Efficient iterative schemes for ab initio total-energy calculations using a plane-wave basis set. Physical Review B 54, 11169 (1996).

18. Perdew, J. P., Burke, K., \& Ernzerhof, M. Generalized gradient approximation made simple. Physical Review Letters 77, 3865 (1996).

19. Blaha, P., Schwarz, K., Madsen, G. K. H., Luitz, D., Lakowski, J., Tran, F., \& Marks, L. An augmented plane wave plus local orbitals program for calculating crystal properties. Vienna Univ. of Technology (2001).

\section{Acknowledgements.}

Crystal growth and properties characterization work at George Mason University was supported by the U.S. Department of Energy, Office of Science, Basic Energy Sciences, Materials Science and Engineering Division. I.I.M. acknowledges support from the U.S. Department of Energy through the grant \#DE-SC0021089. M.P.G. acknowledges the Alexander von Humboldt Foundation, Germany for the equipment grants. Access to the NG-7 SANS instrument was provided by the Center for High Resolution Neutron Scattering, a partnership between the National Institute of Standards and Technology and the National Science Foundation under Agreement No. DMR-2010792. The identification of any commercial product or trade name does not imply endorsement or recommendation by the National Institute of Standards and Technology. We thank J. F. Mitchell for useful 
discussions.

\section{Author contributions.}

N.J.G. and P.E.S. conceived the idea. P.E.S. performed magnetic and magnetotransport measurement and carried out the data analysis. H.B. synthesized single crystals. D.C.J contributed to the figure preparation. R.L.D., L.P. and J.W.L. contributed to neutron diffraction experiments. L.P, N.J.G, J.W.L and M.B. performed SANS experiment. I.I.M. and M.P.G. contributed to the DFT calculations. P.E.S. and N.J.G. wrote the manuscript. All authors contributed to the discussion of the results.

Competing interests: The authors declare no competing interests. 

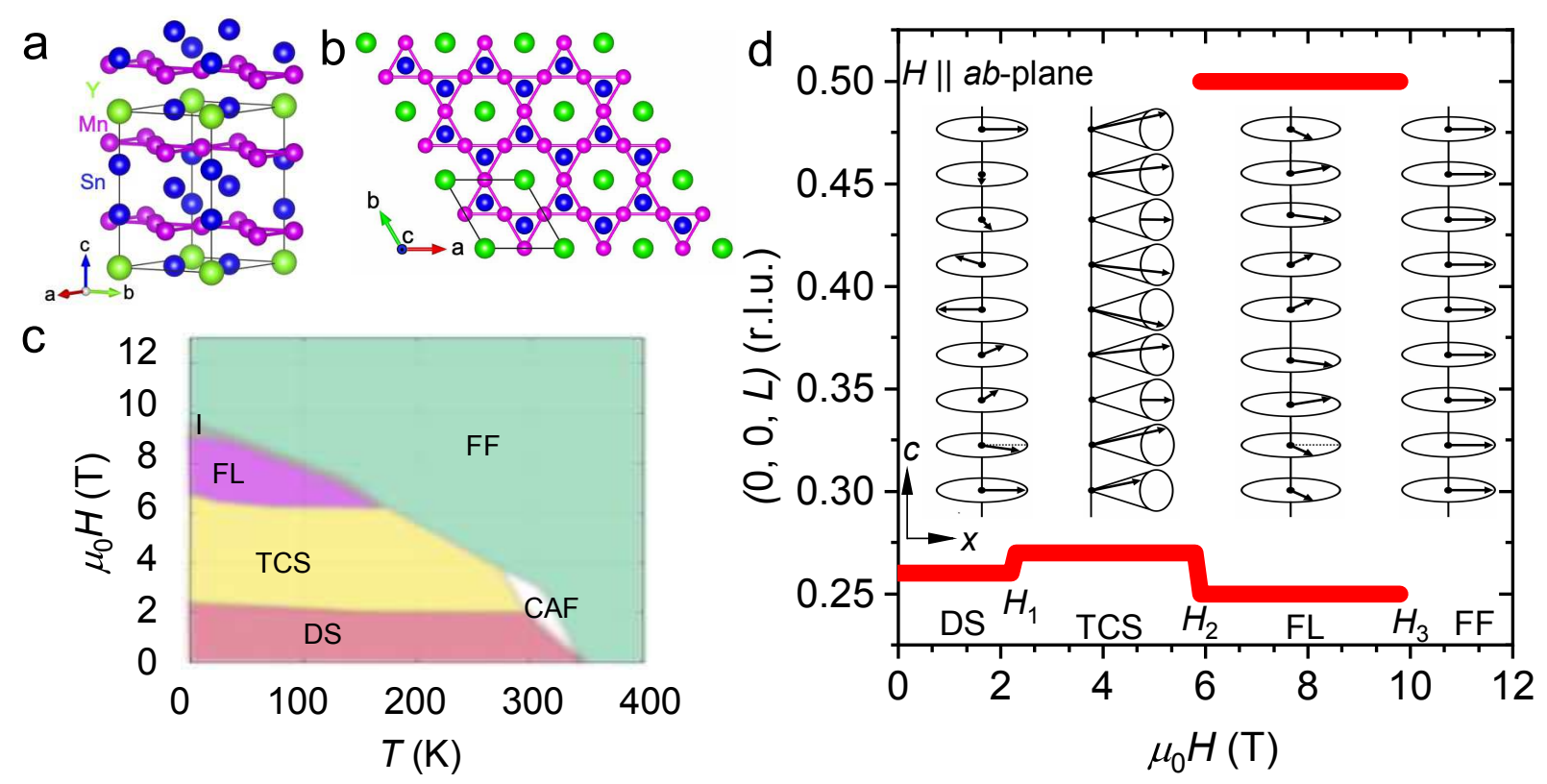

Figure 1: Crystal structure and magnetic properties of $\mathbf{Y M n} \mathbf{S n}_{6}$ • (a) Crystal structure of $\mathrm{YMn}_{6} \operatorname{Sn}_{6}$.

(b) $c$-axis view of the structure showing $\mathrm{Mn}$ atoms arranged in a kagome-net in the $a b$-plane. (c) Schematic $T-H$ phase diagram of $\mathrm{YMn}_{6} \mathrm{Sn}_{6}$ for the magnetic field applied in the $a b$-plane. (d) Plot of the wavevector $\left(k_{z}\right)$ of the magnetic Bragg peaks observed in neutron diffraction experiments (red lines) at $10 \mathrm{~K}$ and illustrations of the magnetic structures of DS, TCS, FL, and FF phases as discussed in the text. Arrows represent the direction of spins in each Mn-layer. Small pockets labelled as I and CAF in panel (c) represent phase 1 and the canted antiferromagnetic phase, respectively. The labels $c$ and $x$ represent $c$-axis and the direction of magnetic field in the $a b$-plane. 

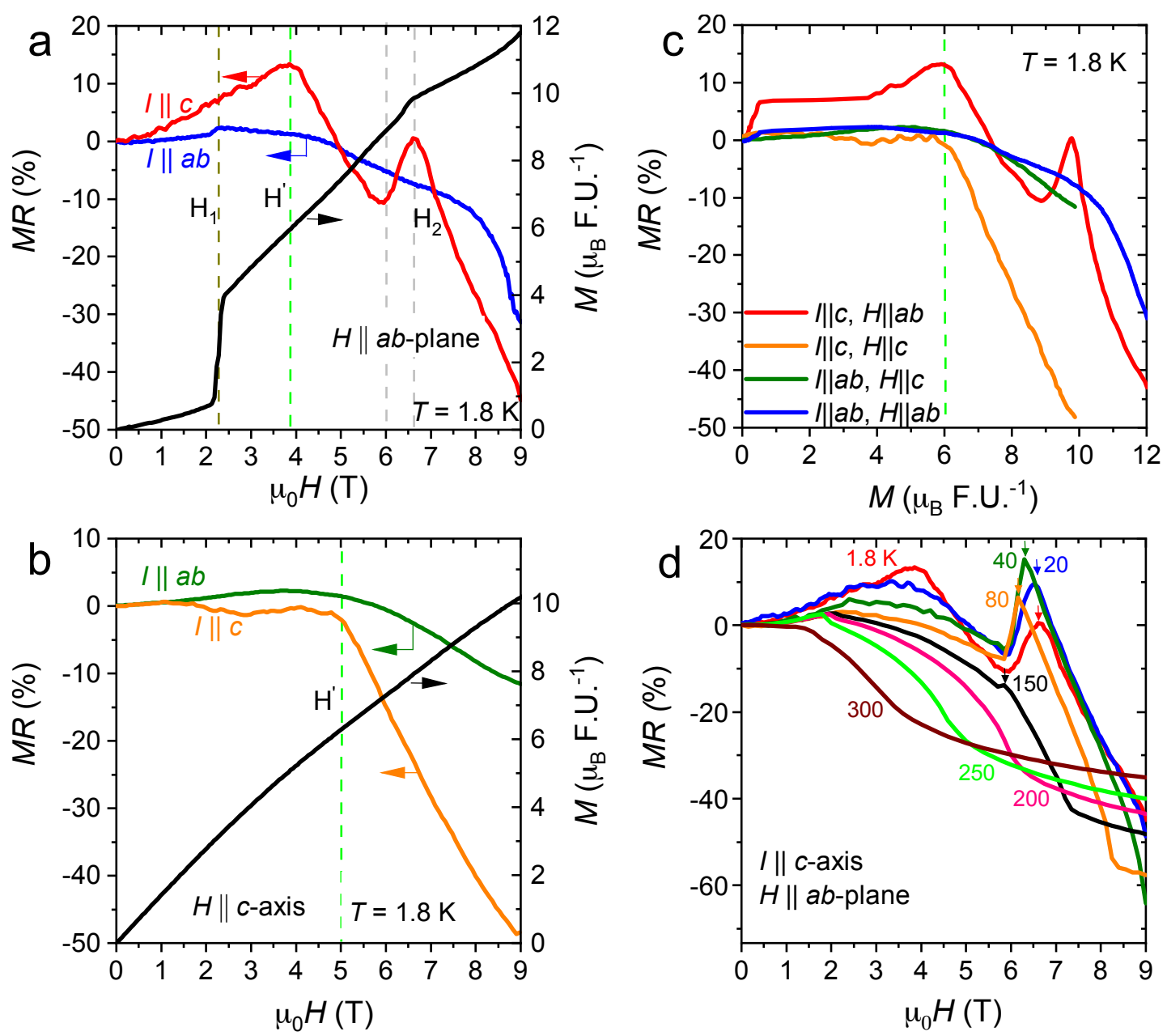

Figure 2: Magnetoresistance, and magnetization of $\mathbf{Y M n}_{6} \mathbf{S n}_{6} \cdot(\mathrm{a}-\mathrm{b}) \mathrm{MR}$ at $1.8 \mathrm{~K}$ as a function of $H$ for $I$ along the $c$-axis and in the $a b$-plane (left axis), and magnetization (right axis). $H$ is either applied in the $a b$-plane, as in panel (a), or along the $c$-axis, as in panel (b). (c) MR as a function of magnetization at $1.8 \mathrm{~K}$ for the directions of $I$ and $H$ indicated in the legend. Demagnetization considerations are discussed in SI1. (d) MR for $I$ along the $c$-axis at the indicated temperatures. The arrows track the MR peak at $\mathrm{H}_{2}$. 

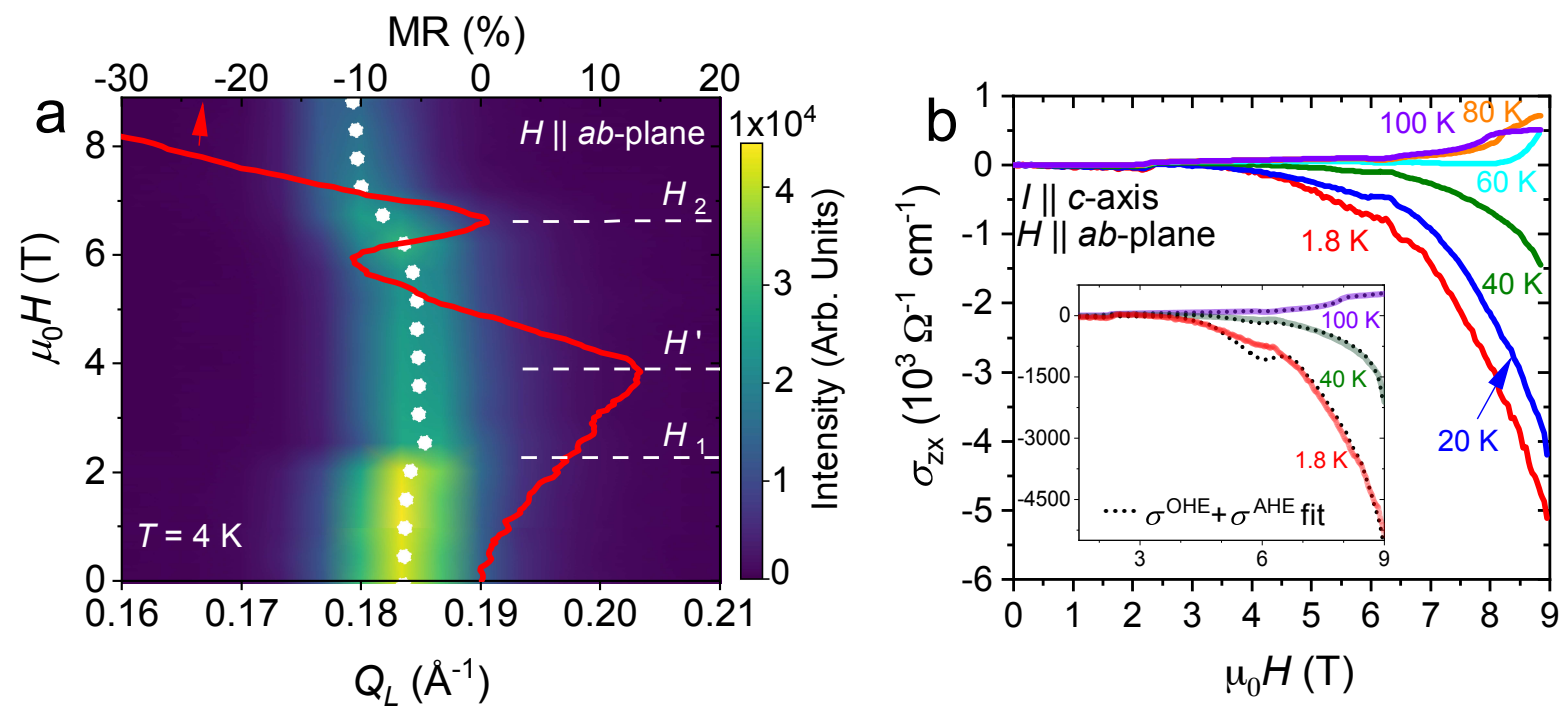

Figure 3: Magnetotransport and SANS intensity of $\mathbf{Y} \mathbf{M n}_{6} \mathbf{S n}_{6}$. (a) Intensity- $Q$ map of the magnetic field dependence of the magnetic Bragg peak observed in small angle neutron scattering [lower axis, modified from ref. (10)] plotted together with the magnetic field dependence of MR for current along the $c$-axis. In both cases, magnetic field is applied in the $a b$-plane. The white dots are a guide to the peak center. (b) Hall conductivity as a function of temperature at indicated temperatures. Inset depicts the fits (dotted lines) of the Hall conductivity comprised of only ordinary, $\sigma^{O H E}=R_{H} H \rho_{z z}^{-2}$, and anomalous, $\sigma^{A H E}=S_{H} M$, contributions. 

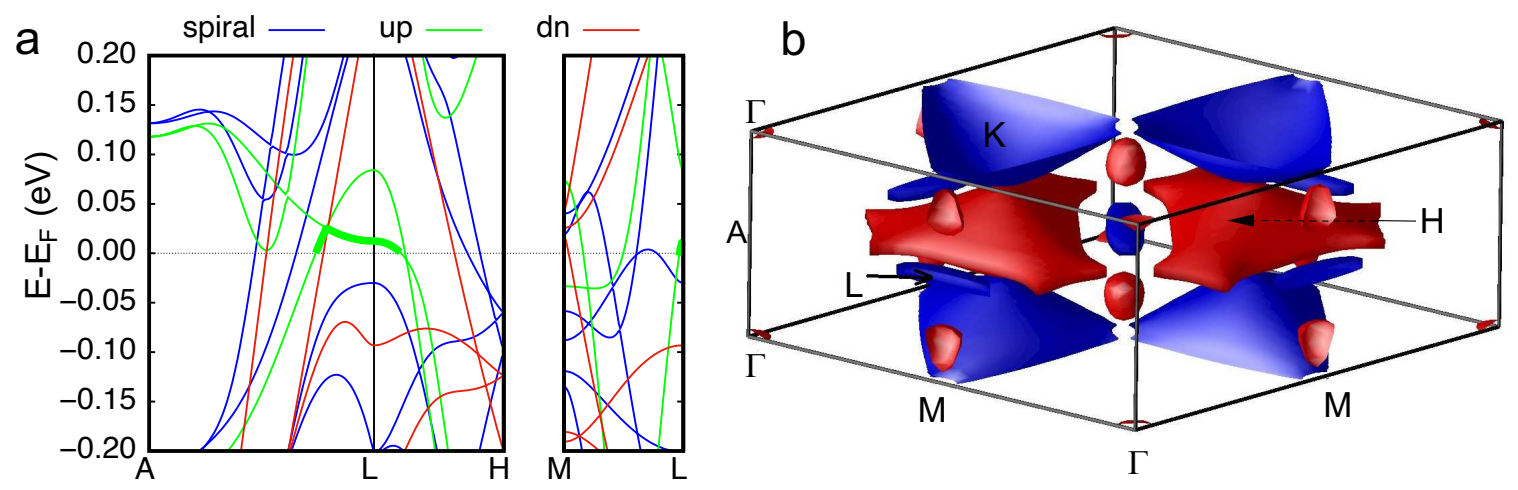

Figure 4: Electronic band structure and Fermi surface of $\mathbf{Y M n}_{6} \mathbf{S n}_{6}$. (a) Calculated electronic band structure at the $L$ point of $\mathrm{YMn}_{6} \mathrm{Sn}_{6}$ in the magnetic spiral ground state, (blue lines) and in the spin polarized state. The lines with green, and red color represent spin up (up) and spin down (dn) bands, respectively. All calculated bands are shown in Fig. SI9. (b) Calculated Fermi surface around $\Gamma, M$ and $L$ points. 


\section{Supplementary Files}

This is a list of supplementary files associated with this preprint. Click to download.

- YMn6Sn6NatComSupplementallnformation.pdf 